\title{
New Approach Based on Texture and Geometric Features for Text Detection
}

\author{
Hinde Anoual ${ }^{1}$, Sanaa El Fkihi ${ }^{1,2}$, Abdelilah Jilbab ${ }^{1,3}$, and Driss Aboutajdine ${ }^{1}$ \\ 1 LRIT, unité associée au CNRST, FSR, Mohammed V University Agdal, Morocco \\ hindanoual@yahoo.fr, aboutaj@fsr.ac.ma \\ 2 ENSIAS, Mohammed V University Soussi, BP 713, Rabat, Morocco \\ elfkihi@ensias.ma \\ 3 ENSET, Madinat AL Irfane, B.P 6207 Rabat-Instituts, Rabat, Morocco \\ jilbab@enset-rabat.ac.ma
}

\begin{abstract}
Due to the huge amount of data carried by images, it is very important to detect and identify the text region as accurately as possible before performing any character recognition. In this paper we describe a text detection algorithm in complex background. It is based on texture and connected components analysis. First we abstract texture regions which usually contain text. Second, we segment the texture regions into suitable objects; the image is segmented into three classes. Finally, we analyze all connected components present in each binary image according to the three classes with the aim to remove non-text regions. Experiments on a benchmark database show the advantages of the new proposed method compared to another one. Especially, our method is insensitive to complex background, font size and color; and offers high precision (83\%) and recall $(73 \%)$ as well.
\end{abstract}

Keywords: Text detection, text localization, feature extraction, texture analysis, geometric analysis.

\section{Introduction}

Text detection is defined as the task that localizes text in complex background without recognizing individual characters. It is still an interesting research topic in many fields. The rational behind this is the fact that, in a given image, the embedded texts are considered as reliable sources of descriptive information since they carry important information on the semantics of the image content. However, there are mainly three text detection challenges: complex background, size of characters, and multiple colors/font styles. To face these challenges, extensive efforts have been made to extract text from images. The existing approaches are based on the mainstream text characteristics that are:

- Texture characteristics: Text region is considered as a texture region to isolate from the rest of the image. There are many kinds of text texture characteristics such as contrast and color homogeneity. Indeed, text must be readable, and that is why the contrast of text is important and highest compared to other objects. Also, characters tend to have the same or similar 
colors. We can found monochrome and polychrome characters in an image. But polychrome characters are related to artistic aspects more than to informative purposes, that is why most of authors tend to discard them.

- Geometric aspects: Text can appear with different sizes. Its size can vary a lot, but this variability must be reasonable. Hence character has size restrictions. In fact, it is not as large as the whole screen, nor smaller than a certain number of pixels. The minimal height and width allowing viewers to read the text are approximately 15 and 7 pixels, respectively. In addition, there is a relationship between character neighbors. Characters in a text have a uniform distance between them.

These two features depend on the type of text. Basically, there are two kinds of text: scene text and artificial text. Scene text appears as a part of and was recorded with the scene like texts in T-shirts and names of streets. Whereas artificial text was produced separately from the image and is laid over the image in a post-processing stage, like text in logos and text for describing images. Here, we focus on the two text kinds.

In this paper we introduce a new efficient text detection technique. Our approach consists of three main steps. In the first stage we aim to abstract texture regions while in the second one, we segment these regions into suitable objects; especially the image is segmented into three classes. In the third step, we analyze all connected components present in each binary image according to the defined classes of the segmented zones.

The remain of this paper is organized as follows: In sections 2 we present a related work to text detection. Section 3 details our new proposed approach. Then, section 4 is devoted to experimental results obtained by comparing our approach to the Y. LIU [1] one of, on the ICDAR 2003 Robust Reading dataset [2. Finally, conclusions are drawn in section 5 .

\section{Related Works}

Numerous text detection algorithms have been proposed in the past years. They are based on different text properties. Moreover, the most feature used in the literature is edge (gradient). For example, Wu et al 3] propose an algorithm based on the image gradient produced by nine second order gaussian derivatives. The pixels that have large gradient are considered as strokes. Then, these strokes are grouped into text blocks based on several empirical rules. Chen et al. 4] and Ye et al. [5] use the canny edge feature and morphological 'close' operation to detect candidate text blocks. In [6], Smith et al. detect text by finding text box 'horizontal rectangle structure of clustered sharp edges', but only text with certain font-size can be detected. Zhong et al [7] proposed a classical text detection algorithm based on connected component analysis. They extract text as those connected components that follow certain size and horizontal alignments constraints. Jain and $\mathrm{Yu}[8]$ use connected component analysis as well. In their algorithm, they decompose the image into a multi-value map, then color connected components are selected. The lasts are determined as text regions if they 
are accepted by any of the following strategies: (1) inter-component features (geometry and connection property), (2) projection profile features in both horizontal and vertical orientations. The probability of missing text is minimized at the cost of increasing false alarms.

Texture analysis is as well a way used to discriminate between text areas and non-text areas. Indeed, techniques based on Gabor filters, Wavelet, Fast Fourier Transform (FFT), spatial variance, etc. can be used to detect the textural properties of a text region in an image. For example, in [9], Li try to capture the texture by mean, second and third order central moments in Haar-wavelet domain. Zhong et al. 10 detect text in JPEG/MPEG compressed domain using texture features from DCT coefficients. They first detect blocks of high horizontal spatial intensity variation as text candidates, and then refine these candidates into regions by spatial constraints. The potential caption text regions are verified by the vertical spectrum energy. But its robustness in complex background may not be satisfying for the limitation of spatial domain features. Kim et al. [11] and Li et al. 12 propose a texture based method using support vector machine (SVM). Another method based on color, texture and OCR statistic features to discriminate texts from non-texts patterns is described in [13.

We the aim to deal with the text detection problem, we propose to capitalize on the texture and geometric aspects of text regions. In the next we will describe our proposed approach.

\section{Proposed Text Detection Approach}

In this section, we present new approach to deal with the text detection problem. The proposed method is mainly based on texture and geometric features, and it consists of three steps that are: Texture Analysis Step, Segmentation Step, and Geometry Analysis one. In the next we will details each step.

\subsection{Texture Analysis Step}

Texture analysis step aims to extract texture regions and filter out smooth ones. Indeed, in this step we divide a grayscale image $I_{m}$ into two kinds of regions: smooth and texture. Then, we decompose an image into $N$ Macro Blocks (MB); the size of the MB was sets to $64 \mathrm{x} 64$ (the size is chosen experimentally). For each $\mathrm{MB}$, the average edge intensity is considered. We adopt the gradient magnitude to obtain the edge image. Let $M B(X, Y)$ denotes the MB at location $(X, Y)$ in an image. The edge intensity of the $M B(X, Y)$ is calculated by:

$$
M B I(X, Y)=\Sigma_{(i, j) \in M B(X, Y)} V(i, j)
$$

Where $V(i, j)$ is the magnitude of the intensity gradient $(I(i, j))$ at location $(\mathrm{i}, \mathrm{j})$, using finite extent convolution masks to estimate the partial derivative. With:

$$
V(i, j)=\sqrt{\left(\frac{d I(i, j)}{d x}\right)^{2}+\left(\frac{d I(i, j)}{d y}\right)^{2}}
$$



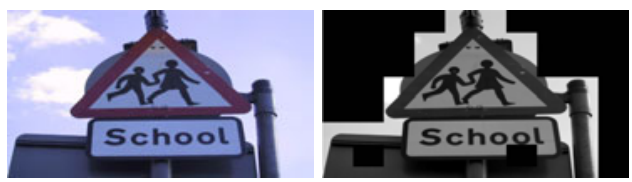

Fig. 1. Color image and its texture analysis result

A MB must have larger edge intensity than the threshold $T_{v}$ to be classified as texture MB. Otherwise, it belongs to smooth regions. $T_{v}$ is determined by:

$$
T_{v}=\alpha \Sigma_{M B(X, Y) \in I_{m}} M B I(X, Y) / N
$$

where $\alpha$ is the weighting factor. Its value has been experimentally sets to 0.75 .

Fig 1 illustrates an input and its outputs of our texture analysis process. The output image is gray level one such that the black regions represent smooth regions and the rest represent texture regions.

\subsection{Segmentation Step}

The segmentation results of texture regions have potential possibilities to improve the text detection results. Thus, applying an enhancement technique on texture regions can benefit the regions segmentation to improve the binarization before employing connected components analysis.

Fig 2 describes a multi-segment decomposed image approach that we propose for segmenting the texture regions. In this approach, we apply a segmentation algorithm which classifies the pixels into $\mathrm{K}$ classes. Then, for each class, we generate the binary image by assuming that this class corresponds to text one. Finally, we analyze the connected components present in each binary image, separately. Hence, for $\mathrm{K}$ classes, we get $\mathrm{K}$ segmented images. The choice of $\mathrm{K}$ is an important and difficult issue. By running several experimentations on our database, we noted that if $\mathrm{K} \leq 2$, a character may be merged with other objects around it and if $K \geq 4$ it may be divided to many components represented in different binary images. So the optimal value of $\mathrm{K}$ is 3 . The Expectation and Maximization method is the segmentation method used here.

As shown in Fig 2, each coherent region is labelled by a color to discriminate regions (yellow, blue and white). The black region of each image represents the smooth one that we are not interested by. After that we represent each coherent region in a single binary image (mask) which will be analyzed in the next step.

\subsection{Geometry Analysis Step}

Text can appear in a single image with different texture. Hence text components can be defined by different coherent regions. Consequently, a totality of text may not be found in a single mask obtained in the previous step. For this reason, we propose to analyze the different components of each mask from the three 


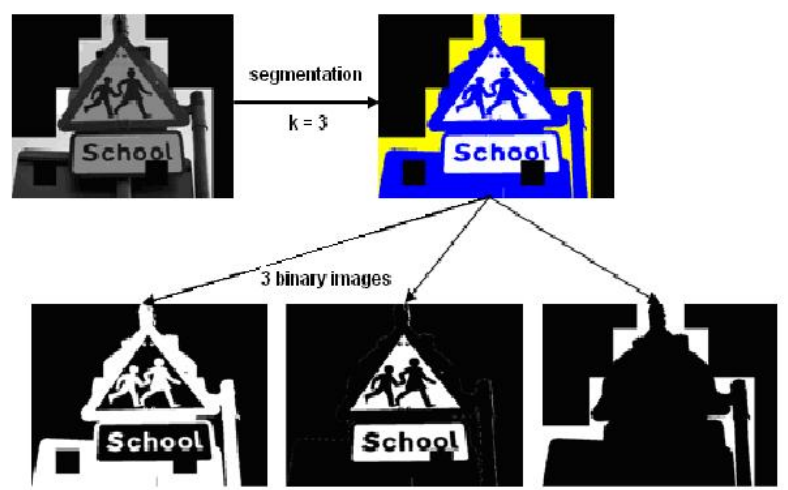

Fig. 2. Segmentation results of the multi-segment decomposed images

obtained ones. Thus, we capitalize on the fact that a text area is a connected component. Nevertheless, the connected components present in an image may include non-text object that are false positives alarms in our case. To deal with this, we propose to use some rules to discriminate text and non-text regions. The used rules are those used in many other text detection published methods such as [5] and [14], but adapted to our case. These rules are related to the geometric and the spatial properties of a text area in an image.

On the first time, we eliminate false positives alarms by using the geometrical properties of a given connected component $i$. Let $W_{i}$ and $H_{i}$ be the width and height of the possible text block labelled $i$. Then, the aspect ratio $\left(R_{i}\right)$ and the area $\left(A_{i}\right)$ of possible text block labelled $i$, are given by:

$$
R_{i}=\frac{W_{i}}{H_{i}} \quad ; \quad A_{i}=W_{i} * H_{i}
$$

The candidate text block is considered as a text block if we have:

$$
0.1 \leq R_{i} \leq 2 \quad ; \quad 0.1 \leq A_{i}
$$

On the second time, we can consider the geometric and spatial relationship of components. Thus, given two neighboring blocks $\left(x_{i} ; y_{i} ; W_{i} ; H_{i}\right)$ and $\left(x_{j} ; y_{j}\right.$; $\left.W_{j} ; H_{j}\right)$, the vicinity based rules can be defined by Eq, Eq, E, and Eq, 8 , where $\left(x_{i} ; y_{i}\right)$ (resp. $\left(x_{j} ; y_{j}\right)$ ) are the coordinates of the object gravity center of the region labelled $i$ (resp. $j$ ).

$$
\begin{gathered}
0.5 \leq \frac{H_{i}}{H_{j}} \leq 2 \\
\operatorname{dist}\left(x_{i}, x_{j}\right) \leq 0.2 \max \left(H_{i}, H_{j}\right) \\
\operatorname{dist}\left(y_{i}, y_{j}\right) \leq 2 \max \left(W_{i}, W_{j}\right)
\end{gathered}
$$

where $\operatorname{dist}(x, y)$ is the distance between $x$ and $y$. 
The first rule given by Eq $[$ ensures that connected components have similar height. The second Eq7 affirms that the connected components are neighbors in horizontal direction while Eq 8 ensures that the considered components belong to same text line [15. Finally, when the different rules above are verified, we obtain an image in which characters are segmented.

\section{Experimental Results}

We used the dataset made available with the occasion of the ICDAR 2003 Robust Reading Competition [2], to evaluate the performance of the new approach compared to the Y. LIU one [1]. The later is based on the gradient magnitude, the connected components and wavelets. The used benchmark database contains 529 images. The existing images depict one or multiple text areas which have different characteristics. Fig 3 illustrates some text detection results. In fact, this figure presents the outputs of the two compared text detection system. We notice that the detection of the text (red block) is accurate and effective, using the new approach.

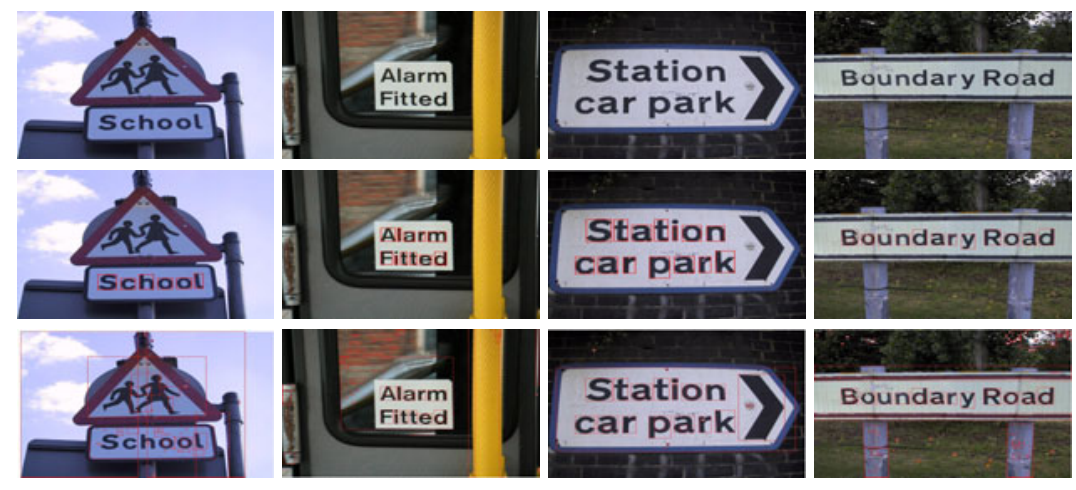

Fig. 3. Results of the compared text detection system. Top: Some inputs. Middle: outputs of our approach. Bottom: outputs of the other method.

For quantitative comparison, two metrics recall and precision are adopted to evaluate the performance of the two compared methods. The recall $(\mathrm{R})$ is the number of correct estimates (C) divided by ground-truth text regions $(\mathrm{T})$ whereas the precision $(\mathrm{P})$ is the ratio of the number of correct estimates $(\mathrm{C})$ to the number of regions claimed (E). These parameters are defined by:

$$
R=\frac{C}{T} \quad ; \quad P=\frac{C}{E}
$$

The highest value of the recall $(\mathrm{R})$ denotes the superior ability in detecting the relevant text region, while the highest value of precision $(\mathrm{P})$ indicates the highest detection rate with correct text regions. 


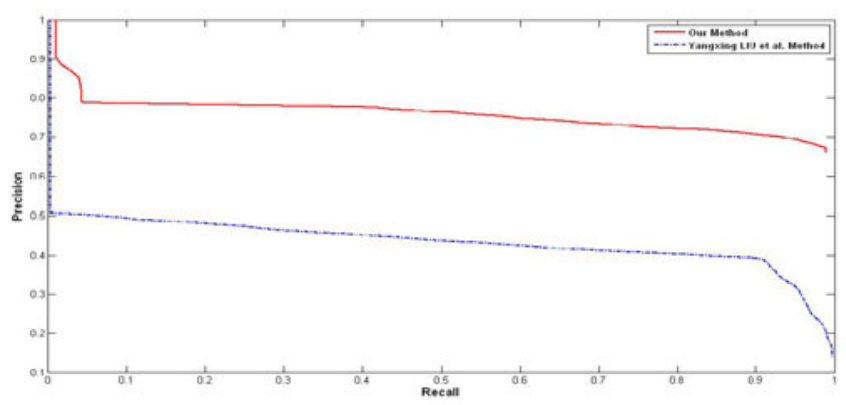

Fig. 4. The Recall/Precision curves of our method and the Y. LIU one

Figure 4 gives the Recall/Precision curves of our method compared to the Y. LIU one [1]. This figure highlights that the new approach offers more precision for all recall values, compared to the other approach. The precision rate is $83 \%$ (resp. 59\%) and the recall rate is $73 \%$ (resp. $73 \%$ ) for the new approach (resp. Y. LIU's method).

In addition, precision and recall can be combined into a single standard measure of quality (f) given by Eq. 10. The relative weights of recall and precision are controlled by $\alpha$ which we set to 0.5 in order to give equal weight to the two considered metrics.

$$
f=\frac{1}{\alpha / P+(1-\alpha) / R}
$$

For our proposed approach, the value of $f$ is equal to 0.78 while it is equal to 0.65 in case of the compared method.

In terms of qualitative and quantitative evaluations, we conclude that the new proposed approach is more efficient and robust compared to the other one.

\section{Conclusion}

This paper describes an automatic text detection method in images with complex background. The proposed approach is basically based on the textured areas contained in an image. Indeed, Texts are considered as textured zones that we have to distinct from a smooth ones. Then, a segmentation method is applied on texture regions with the aim to decompose the texture regions into different coherent regions. The lasts are separately analyzed in order to select the candidate text regions. Finally, The results are combined to define text regions.

Simulation results on the public benchmark ICDAR 2003 Robust Reading Dataset show that our method detects efficiently characters in spite of the complexity of image background. The precision and recall rates are $83 \%$ and $73 \%$ respectively. In addition, the standard measure of quality $\mathrm{f}$ is equal to 0.78 . Consequently, the proposed method is accurate and effective.

In future works, we propose to improve the proposed approach by minimizing the number of holes in case of false detection text alarms. 


\section{References}

1. Liu, Y., Goto, S., Ikenaga, T.: A Contour-Based Robust Algorithm for Text Detection in Color Images. IEICE Transactions 89-D(3), 1221-1230 (2006)

2. http://algoval.essex.ac.uk/icdar/RobustReading.html

3. Wu, V., Manmatha, R., Riseman, E.M.: Finding text in images. In: DL 1997: Proceedings of the second ACM international conference on Digital libraries, New York, NY, USA, pp. 3-12. ACM, New York (1997)

4. Chen, D.T., Bourlard, H., Thiran, J.-P.: Text identification in complex background using SVM. In: International Conference on Computer Vision and Pattern Recognition 2001, pp. 621-626 (2001)

5. Ye, Q., Gao, W., Wang, W., Zeng, W.: A robust text detection algorithm in images and video frames. In: Joint Conference of Fourth International Conference on Information Communications and Signal Processing and Pacific-Rim Conference on Multimedia, Singapore (2003)

6. Smith, M.A., Kanade, T.: Video skimming for quick browsing based on audio and image characterization, Carnegie Mellon University, Pittsburgh, PA, Technical Report CMU-CS-95-186 (July 1995)

7. Zhong, Y., Karu, K., Jain, A.K.: Locating text in complex color images. In: ICDAR '95: Proceedings of the Third International Conference on Document Analysis and Recognition, Washington, DC, USA, vol. 1, p. 146. IEEE Computer Society, Los Alamitos (1995)

8. Jain, A.K., Yu, B.: Automatic text location in images and video frames. Pattern Recognition 31, 2055-2076 (1998)

9. David, H.L., Doermann, D., Kia, O.: Automatic text detection and tracking in digital video. IEEE Transactions on Image Processing 9(1) (2000)

10. Zhong, Y., Zhang, H., Jain, A.K.: Automatic caption localization in compressed video. IEEE Trans. Pattern Anal. Mach. Intell. 22(4), 385-392 (2000)

11. Kim, K.I., Jung, K., Kim, J.H.: Texture-based approach for text detection in image using support vector machine and continuously adaptive mean shift algorithm. IEEE Transaction on PAMI 25, 1631-1639 (2003)

12. Li, X., Wang, W., Jiang, S., Huang, Q., Gao, W.: Fast and effective text detection. In: ICIP, pp. 969-972. IEEE, Los Alamitos (2008)

13. Ye, Q., Jiao, J., Huang, J., Yu, H.: Text detection and restoration in natural scene images. J. Vis. Comun. Image Represent. 18(6), 504-513 (2007)

14. Ezaki, N., Bulacu, M., Schomaker, L.: Text detection from natural scene images: Towards a system for visually impaired persons. In: ICPR (2), pp. 683-686 (2004)

15. Hanif, S., Prevost, L.: Text detection and localization in complex scene images using constrained adaboost algorithm, pp. 1-5 (2009) 